\title{
The role of miRNAs in autoimmune inflammatory diseases
}

\begin{abstract}
Introduction
MicroRNAs (miRNAs) are abundant short 2123 nucleotides-long non-coding RNAs that modulate virtually all eukaryotic gene expression processes during development and are crucial for sustaining optimal cell functions and tissue homeostasis [1]. Therefore the processing and (dys) regulation of the processing of these miRNAs are critical in the pathogenesis of many diseases [2]. Several studies have convincingly demonstrated the relationship of microRNAs expression with nutrition, inflammation and cancer [3-5].
\end{abstract}

This short communication focuses on our understanding about the role of miRNA in the pathogenesis of rheumatic diseases and the possible involvement in accelerated atherogenesis, an aspect that is related to an increased prevalence of coronary artery disease in these patients [6,7]. Recent attention has been given to the pathogenetic connection of miRNAs to metabolic and inflammatory conditions $[4,5]$ which are known to be risk factors for cardiovascular diseases [8].

\section{microRNAs production}

microRNAs have emerged as new class of modulators of gene expression at posttranscriptional level [9]. They are small non-coding molecules of RNA that bind to complementary sequences in the 3' UTR of multiple target mRNAs, usually resulting in their silencing. These sequences are transcribed in the nucleus of eukaryotic cells by either RNA polymerase II or RNA polymerase III into primary miRNA transcripts (pri-miRNA), that are cleaved by the microprocessor complex Drosha-DGCR8 (Pasha) [1,2]. After this cleavage, the resulting precursor hairpin, the pre-miRNA, is exported from the nucleus by Exportin-5-Ran-GTP; once in the cytoplasm, the RNase Dicer complex with TRB protein cleaves the pre-miRNA hairpin to its mature length. Now the mature miRNA is loaded togheter with Argonaute (Ago2) proteins into the RNA-Induced Silencing Complex (RISC) that guides to silencing target mRNA $[1,9]$.
microRNAs appear as important cytoplasmic regulators of gene expression. miRNAs act as post-transcriptional regulators of their messenger RNA targets via mRNA degradation and/or translational repression. However, it is becoming evident that miRNAs also have nuclear functions, including transcriptional control $[1,2]$.

\section{microRNAs and inflammation}

The increased prevalence of cardiovascular mortality rate in rheumatic diseases cannot only be justified by the presence of traditional atherosclerotic risk factors but is strongly associated with a chronic inflammatory response typical of Systemic Lupus Erythematosus (SLE) or Rheumatoid Arthritis (RA) [10-12]. Unrestrained activation of both the innate and the adaptive immune responses, occurring in rheumatic diseases, may in turn explain novel mechanisms in the pathogenesis of atherosclerosis [13].TLR ligand-induced microRNAs, including miR-146a, miR-155 and miR-132, play essential roles in regulating inflammatory mediators, with a strong impact on the immune system [14].

Persistent inflammation is the starring character in chronic immune-mediated rheumatic diseases and it may play an important role in endothelial dysfunction, the first step of atherogenesis [15]. There is a complex system of checkpoints and molecules involved in the regulation of the immune system that prevent the occurrence of disorders like autoimmune diseases and cancer [16] and accumulating evidence suggests that numerous microRNAs are aberrantly expressed in human diseases $[3,5]$.

MicroRNAs have been considered important regulators in both physiological and disease contexts. Among all miRNAs, the miR-34 family (miR-34a, -34b, -34c), which has been well characterized as a tumor suppressor, displays diverse functions in noncancerous diseases [17]. MiR-34 levels are relatively low in the cardiovascular system, but recently they have been reported to function in cardiovascular disorders by regulating apoptosis, telomere attrition, DNA damage, and inflammatory response [18].

\section{Federica Canzano, Alessia Paganelli, Paola Volpe \& Roberto Paganelli*}

Department of Medicine and Sciences of Aging, University "G. d'Annunzio" of ChietiPescara

*Author for correspondence: roberto. paganelli@unich.it 


\section{microRNAs in rheumatoid arthritis (RA)}

Many published studies have uncovered the differential expression of miRNAs and the role they might play in rheumatic diseases [19,20]. Both innate and adaptive immune responses are controlled by activities due to miRNAs production [20,21]. Therefore many cell types, including macrophages and lymphocyte subsets relevant to autoimmune, inflammatory and other rheumatic disorders, are affected and regulated by miRNA expression [22-24].

Rheumatoid arthritis is characterized by polymorphisms of DRB1*0401 (DR 4, Dw 4; in $50 \%$ cases of RA) DRB1*0404 (DR 4 , Dw 14: in $30 \%$ cases of RA) DRB1*0101 (DR 1 , Dw 1 ; in $24 \%$ cases of RA), B-cell proliferation with production of autoantibodies and increased pro-inflammatory and TH1-type cytokines production $[25,26]$.

Specific micro-RNAs are responsible of aberrant expression of Interleukin (IL)-1 and tumor necrosis factor (TNF) a: in synovial fibroblasts, inflammatory cells and Th 17 cells of RA patients [27], miR-146 and miR-155 are up-regulated significantly compared to healthy controls [28]. These two miRNAs have been particularly investigated as potential targets for intervention in RA, since both miR-155 [29] and miR-146a have been shown to be highly expressed in human RA synovial tissue and it is induced by the proinflammatory cytokines TNF $\alpha$ and IL$1 \alpha[30]$. Levels of miR-146, miR-155 and miR16 were found to be upregulated in the peripheral blood of patients with RA [27], and miR-146 and miR-16 levels were higher in patients with active $(n=8)$ rather than inactive $(n=3)$ disease, suggesting that these miRNAs could be potential markers of disease activity [30,31].

\section{miRNAs in other rheumatic diseases}

Recent studies suggest a dysregulation of miRNAs expression in B- and T-lymphocytes of SLE patients [32,33]. In a study of the levels of miR-146a and miR-155 in the serum and urine of patients with SLE Wang et al. [33] observed a downregulation of miR-146 in peripheral blood mononuclear cells, which led to amplify the IFN-pathway causing the stimulation of TRAF-6, IRAK1 and IRF5 and targeting IFN regulatory factor 5 and STAT-1, already known to identify key markers and pathogenetic factors in SLE [34,35].

The experimental inhibition of endogenous miR146a increases the induction of type I IFNs in
PBMCs so it directly represses the transactivation downstream of type I IFN production $[34,36]$. Maintenance of suppressor/regulatory cell activity has been shown to be assured by miR155 [37], which is also able to downregulate lipopolysaccharide-induced inflammatory pathways in human monocyte-derived dendritic cells [23]. The expression of miR-155 has also been shown to correlate with the progression of lung fibrosis in systemic sclerosis patients [38].

The activation of TLR4, TLR2, and TLR5 signaling pathways or stimulation of inflammatory cytokines such as TNFa and IL$1 \beta$ are strongly associated with the upregulation of miR-146a in monocytes [21]. Other miRNAs such as miR-155 were identified as negative regulators of TLR-signaling in response to both bacterial- and viral-derived antigens that activate TLR4, TLR2, TLR3, or TLR9 [39,40].

The aberrant expression of miR-146 and miR155 in rheumatic diseases could underlie the increased cardiovascular mortality rate $[10,19]$. A recent elegant study reveals that miR-146a promotes proliferation and migration of rat vascular smooth muscle cells in vitro in a nuclear factor- $\kappa \mathrm{B}$-dependent manner [41]. Other studies demonstrate that miR-146a inhibits oxidized LDL-induced lipid accumulation and inflammatory response via targeting toll-like receptor 4 [42]. In a closed loop, oxidized LDLs in turn upregulate miR-146a [43].

Stanczyk et al. suggest that miR-155 is responsible for post-trancriptional silencing of Matrix Metallo Peptidases 3 and 1 [28]: this discovery reveals that miR-155 plays an important role both on inflammatory reaction against synovial tissue and in the atherosclerotic plaque.

It also has been found that miR-155 stimulates the differentiation of endothelial cells [44], fibroblasts and the inflammatory dysfunction; miR-146a increases proliferation of fibroblasts and collagen syntesis [31] in many types of arthritis and in other fibrotic conditions. In this respect, miR-24 and other miRNAs exert regulatory functions on chondrocytes and matrix changes in osteoarthritis $[45,46]$ and they also operate in the framework of senescenceassociated remodelling of several pathways affecting many age-related diseases [47].

\section{Conclusions}

MicroRNAs have been linked to many human diseases and are being pursued as clinical diagnostics and as therapeutic targets $[1,4,5]$. 
More than 2000 miRNAs have been identified and it is believed that they collectively regulate one third of the genes in the human genome [48]. The potential of miRNAs as biomarkers and/or therapeutic targets in rheumatic diseases is a new area of research [19] for which currently available data are promising and expanding but still limited.

\section{Conflict of interest}

The Authors declare no conflict of interest for the subject of this manuscript.

\section{References}

1. Bartel DP. MicroRNAs: Genomics, biogenesis, mechanism, and function. Cell. 116(2), 281-297 (2004).

2. Newman MA, Hammond SM. Emerging paradigms of regulated microRNA processing. Genes. Dev. 24(11), 1086-1092 (2010).

3. Schmittgen TD. Regulation of microRNA processing in development, differentiation and cancer. J. Cell. Mol. Med. 12(5b), 1811-1819 (2008).

4. Acunzo M, Croce CM. MicroRNA in Cancer and Cachexia-A Mini-Review. J. Infect. Dis. 212(suppl 1), S74-S77 (2015).

5. Cui J, Zhou B, Ross SA et al. Nutrition, microRNAs, and Human Health. Adv. Nutr. 8(1), 105-112 (2017).

6. Gonzalez-Juanatey C, Gonzalez-Gay MA. Rheumatoid arthritis and accelerated atherogenesis. Circulation. 109(25), e328 (2004).

7. Mackey RH, Kuller LH, Moreland LW. Cardiovascular disease risk in patients with rheumatic diseases. Clin. Ger. Med. 33(1), 105117 (2017).

8. Tousoulis D, Kampoli AM, Papageorgiou N et al. Pathophysiology of atherosclerosis: The role of inflammation. Curr. Pharm. Des. 17(37), 40894110 (2011).

9. Yates LA, Norbury CJ, Gilbert RJC. The long and short of microrna. Cell. 153(3), 516-519 (2013).

10. Lopez-Mejas R, Castaneda S, Gonzalez-Juanatey $\mathrm{C}$ et al. Cardiovascular risk assessment in patients with rheumatoid arthritis: The relevance of clinical, genetic and serological markers. Autoimmun. Rev. 15(11), 1013-1030 (2016).

11. Croca S, Rahman A. Atherosclerosis in systemic lupus erythematosus. Best. Pract. Res. Clin. Rheumatol. 31(3), 364-372 (2017).

12. Gonzalez-Gay MA, Gonzalez-Juanatey C, Martin J. Rheumatoid arthritis: A disease associated with accelerated atherogenesis. Semin. Arthritis.
Rheum. 35(1), 8-17 (2005).

13. Kerekes G, Soltasz P, Nurmohamed MT et al. Validated methods for assessment of subclinical atherosclerosis in rheumatology. Nat. Rev. Rheumatol. 8(4), 224-234 (2012).

14. Nahid MA, Satoh M, Chan EKL. MicroRNA in TLR signaling and endotoxin tolerance. Cell. Mol. Immunol. 8(5), 388-403 (2011).

15. Gimbrone MA Jr, Garcia-Cardena G. Endothelial cell dysfunction and the pathobiology of atherosclerosis. Circ. Res. 118(4), 620-636 (2016).

16. Dyck L, Mills KHG. Immune checkpoints and their inhibition in cancer and infectious diseases. Eur. J. Immunol. 47(5), 765-779 (2017).

17. Rokavec M, Li H, Jiang L et al. The p53/miR34 axis in development and disease. J. Mol. Cell. Biol. 6(3), 214-230 (2014).

18. Li N, Wang K, Li PF. MicroRNA-34 family and its role in cardiovascular disease. Crit. Rev. Eukaryot. Gene. Expr. 25(4), 293-297 (2015).

19. Nakasa T, Nagata Y, Yamasaki K et al. A minireview: microRNA in arthritis. Physiol. Genomics. 43(10), 566-570 (2011).

20. Luo X, Ranade K, Talker R et al. microRNAmediated regulation of innate immune response in rheumatic diseases. Arthritis. Res. Ther. 15(2), 210 (2013).

21. Taganov KD, Boldin MP, Chang KJ et al. NFkappaB-dependent induction of microRNA miR-146, an inhibitor targeted to signaling proteins of innate immune responses. Proc. Natl. Acad. Sci. USA. 103(33), 12481-12486 (2006).

22. Baltimore D, Boldin MP, O'Connell RM et al. MicroRNAs: new regulators of immune cell development and function. Nat. Immunol. 9(8), 839-845 (2008).

23. Ceppi M, Pereira PM, Dunand-Sauthier I et al. MicroRNA-155 modulates the interleukin-1 signaling pathway in activated human monocytederived dendritic cells. Proc. Natl. Acad. Sci. USA. 106(8), 2735-2740 (2009).

24. Danger R, Braza F, Giral M et al. MicroRNAs, major players in B cells homeostasis and function. Front. Immunol. 5, 98 (2014).

25. Roudier J. Association of MHC and rheumatoid arthritis. Association of RA with HLA-DR4: The role of repertoire selection. Arthritis. Res. 2, 217-220 (2000).

26. Goronzy JJ, Weyand CM. Rheumatoid arthritis. Immunol. Rev. 204(1), 55-73 (2005).

27. Pauley KM, Satoh M, Chan AL et al. Upregulated miR-146a expression in peripheral blood mononuclear cells from rheumatoid arthritis 
patients. Arthritis. Res. Ther. 10(4), R101 (2008).

28. Stanczyk J, Pedrioli DM, Brentano F et al. Altered expression of MicroRNA in synovial fibroblasts and synovial tissue in rheumatoid arthritis. Arthritis. Rheum. 58(4), 1001-1009 (2008).

29. Su LC, Huang AF, Jia $\mathrm{H}$ et al. Role of microRNA-155 in rheumatoid arthritis. Int. J. Rheum. Dis. 20(11), 1631-1637 (2017).

30. Nakasa T, Miyaki S, Okubo A et al. Expression of microRNA-146 in rheumatoid arthritis synovial tissue. Arthritis. Rheum. 58(5), 1284-1292 (2008).

31. Saferding V, Puchner A, Goncalves-Alves E et al. MicroRNA-146a governs fibroblast activation and joint pathology in arthritis. J. Autoimmun. 82, 74-84 (2017).

32. Te JL, Dozmorov IM, Guthridge JM et al. Identification of unique microRNA signature associated with lupus nephritis. PLoS. One. 5(50, e10344 (2010).

33. Wang G, Tam LS, Kwan BC et al. Expression of miR-146a and miR-155 in the urinary sediment of systemic lupus erythematosus. Clin. Rheumatol. 31(3), 435-40 (2012).

34. Smith S, Fernando T, Wu PW et al. MicroRNA302d targets IRF9 to regulate the IFN-induced gene expression in SLE. J. Autoimmun. 79, 105111 (2017).

35. Oon S, Wilson NJ, Wicks I. Targeted therapeutics in SLE: emerging strategies to modulate the interferon pathway. Clin. Transl. Immunology. 5(5), e79 (2016).

36. Park H, Huang X, Lu C et al. MicroRNA-146a and microRNA-146b regulate human dendritic cell apoptosis and cytokine production by targeting TRAF6 and IRAK1 proteins. J. Biol. Chem. 290(5), 2831-2841 (2015).

37. Lu LF, Thai TH, Calado DP et al. Foxp3dependent microRNA155 confers competitive fitness to regulatory $\mathrm{T}$ cells by targeting SOCS1 protein. Immunity. 30(1), 80-91 (2009).

38. Christmann RB, Wooten A, Sampaio-Barros P et al. miR-155 in the progression of lung fibrosis in systemic sclerosis. Arthritis. Res. Ther. 18(1), 155 (2016).

39. O'Connell RM, Taganov $\mathrm{KD}$, Boldin $\mathrm{MP}$ et al. MicroRNA-155 is induced during the macrophage inflammatory response. Proc. Natl. Acad. Sci. USA. 104(5), 1604-1609 (2007).

40. Tili E, Michaille JJ, Cimino A et al. Modulation of miR-155 and miR-125b levels following lipopolysaccharide/TNF-alpha stimulation and their possible roles in regulating the response to endotoxin shock. J. Immunol. 179(8), 50825089 (2007).

41. Luo Y, Xiong W, Dong S et al. MicroRNA-146a promotes the proliferation of rat vascular smooth muscle cells by downregulating p53 signaling. Mol. Med. Rep. 16(5), 6940-6945 (2017).

42. Yang K, He YS, Wang XQ et al. MiR-146a inhibits oxidized low-density lipoproteininduced lipid accumulation and inflammatory response via targeting toll-like receptor 4. FEBS. Lett. 585(6), 854-860 (2011).

43. Li Z, Wang S, Zhao W et al. Oxidized lowdensity lipoprotein upregulates microRNA-146a via JNK and NF-кB signaling. Mol. Med. Rep. 13(2), 1709-1716 (2016).

44. Yang D, Wang J, Xiao M et al. Role of Mir155 in controlling HIF-1 $\alpha$ level and promoting endothelial cell maturation. Sci. Rep. 6(1), 35316 (2016).

45. Philipot D, Guérit D, Platano D et al. p16INK4a and its regulator miR-24 link senescence and chondrocyte terminal differentiation-associated matrix remodelling in osteoarthritis. Arthritis. Res. Ther. 16(1), R58 (2014).

46. Sondag GR, Haqqi TM. The role of micrornas and their targets in osteoarthritis. Curr. Rheumatol. Rep. 18(8), 56 (2016).

47. Munk R, Panda AC, Grammatikakis I et al. Senescence-associated micrornas. Int. Rev. Cell. Mol. Biol. 334, 177-205 (2017).

48. Hammond SM. An overview of microRNAs. Adv. Drug. Deliv. Rev. 87, 3-14 (2015). 